\title{
Optimization of Multi-Query based on Heuristic Approach
}

\author{
Prof. A. D. Isalkar \\ Department of IT, RTMNU, DMIETR, Wardha, Maharashtra, India
}

\begin{abstract}
Now a day, it is very common to see that complex queries are being vastly used in the real time database applications. These complex queries often have a lot of common sub-expressions, either within a single query or across multiple such queries run as a batch. Multi-query optimization aims at exploiting common sub-expressions to reduce evaluation cost. Multi-query optimization has often been viewed as impractical, since earlier algorithms were exhaustive, and explore a doubly exponential search space. This work demonstrates that multi-query optimization using heuristics is practical, and provides significant benefits. The cost-based heuristic algorithms: basic Volcano-SH and Volcano-RU, which are based on simple modifications to the Volcano search strategy. The algorithms are designed to be easily added to existing optimizers. The study shows that the presented algorithms provide significant benefits over traditional optimization, at a very acceptable overhead in optimization time.
\end{abstract}

Keywords: Heuristics, Volcano-SH and Volcano-RU.

\section{INTRODUCTION}

The main idea of Multi-Query Optimization is to optimize the set of queries together and execute the common operation once. Complex queries are becoming commonplace, with the growing use of decision support systems.

\section{Approaches to Query Optimization: \\ - $\quad$ Systematic query optimization. \\ - $\quad$ Heuristic query optimization. \\ - Semantic query optimization.}

1.1 Systematic query optimization

In systematic query optimization, the system estimates the cost of every plan and then chooses the best one. The best cost plan is not always universal since it depends on the constraints put on data. For example, joining on a primary key may be done more easily than joining on a foreign key since primary keys are always unique and therefore after getting a joining partner, there is no other key expected. The system therefore breaks out of the loop and hence does not scan the whole table. Though in many cases efficient, it is a time wasting practice and therefore sometimes it can be done away with .The costs considered in systematic query optimization include access cost to secondary storage, storage cost, computation cost for intermediate relations and communication costs.

\section{$1.2 \quad$ Heuristic query optimization}

In the heuristic approach, the operator ordering is used in a manner that economizes the resource usage but conserving the form and content of the query output. The principle aim is to:

(i) Set the size of the intermediate relations to the minimum and increase the rateat which the intermediate relation size tend towards the final relation so as to optimize memory.

(ii) Minimize on the amount of processing that has to be done on the data without affecting the output

\subsection{Semantic query optimization:}

This is a combination of Heuristic and Systematic optimization. The constraintsspecified in the database schema can be used to modify the procedures of the heuristicrules making the optimal plan selection highly creative. This leads to heuristicrules that are locally valid though cannot be taken as rules of the thumb.

\section{MULTI QUERY PROCESSING}

In multi query optimization, queries are optimized and executed in batches. Individualqueries are transformed into relational algebra expressions and are representedas graphs the graphs are created in such away that:

(i) Common sub-expressions can be detected and unified; 
(ii) Related sub-expressions are identified so that the more encompassing sub expressionis executed and the other subexpressions are derived from it. Before studying how exactly optimizer works, or how exactly the optimization takes place, we need to first understand where this phase of optimization is implemented in the execution of a query. For that we have to take a brief look on query processing.

Query processing refers to the range of activities involved in extracting the data from database. The activities include transformation of queries in high level database languages into expressions that can be used at physical level of the file system, a variety of query optimizing transformations and actual evaluation of queries.

Referring to the fig the basic steps involved in the query processing are

1) Parsing and translation

2) Optimization

3) Evaluation

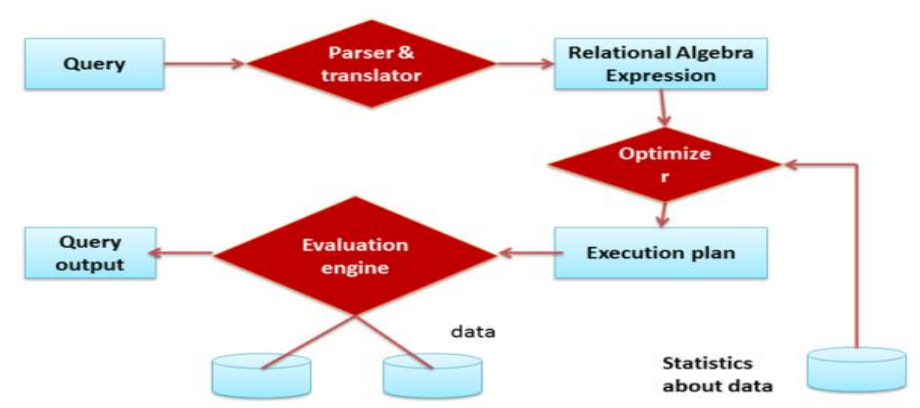

Figure 1: Query Processing

Before query processing can begin, the system must translate a query into a usable form. A language such as SQL is suitable for human use, but is ill-suited to be the system's internal representation of a query. A more suitable internal representation is one based on the extended relational algebra.

Thus, the first action the system must take in query processing is to translate a given query in its internal form. This translation process is similar to the work performed by the parser of a compiler. In generating the internal form of the query, the system uses parsing techniques. The parser used in this query checks the syntax of the query. It verifies that the names of relations appearing in the query as those really present in the database and so on. Thus after constructing the parse tree representation of a query, it translates into the relational algebra expression.

After this, the optimizer comes into play. It takes the relational algebra expression as the input from parser. By applying a suitable optimizing algorithm, it finds out the best plan among the various plans possible. The best plan is nothing but the plan requiring minimum cost for its execution. This plan is provided as the input for further processing.

Finally, the query evaluation engine takes this plan as input, executes it and returns the output of the query. The output is nothing but the specified number of tuples satisfying that query.

Thus, the above figure represents exact location in the whole query processing where the optimizer works.

Generation of optimal global queries is not necessarily done on individual optimalplans. It is done on the group of them. This leads to a large sample space fromwhich the composite optimal plan has to be got. For example, if for four relations $\mathrm{A}, \mathrm{B}, \mathrm{C}$, and $\mathrm{D}$ there are two queries whose optimal states are Q1 $=(\mathrm{A} \bowtie \mathrm{B}) \bowtie \mathrm{C}$ and $\mathrm{Q} 2=(\mathrm{B} \bowtie \mathrm{C}) \bowtie \mathrm{D}$ with execution costs e1 and e2 respectively, the total cost is e1 + e2.Though these queries are individually optimal, the sum is not necessarily optimal.The query Q1 for example can be rearranged to an individually non optimal stateQ' $=$ A $(\mathrm{B} \bowtie \mathrm{C})$ whose cost, say E1 is greater than e1. The combination of Q' and Q2may make a more optimal plan globally at run time in case they cooperate. Sincethere is a common expression (BळC), it can be executed once and the result sharedby the two queries. This leads to a cost of E1+ e2 -E2where E2 is the cost of evaluating $(\mathrm{B} \bowtie \mathrm{C})$ which can be less than $\mathrm{e} 1+\mathrm{e} 2$. If sharing was tried on individual optimalplans, sharing would be impossible hence the saving opportunity would be lost. Toachieve cost savings using sharing, both optimal and non-optimal plans are neededso that the sharing possibilities are fully explored. This however increase the samplespace for the search hence a more search cost. The search strategy therefore needsto be efficient enough to be cost effective. Though this approach can lead to a lotof improvement on the efficiency of a query, it may still have some bottlenecks thathave to be overcome if a global state is at all times to be achieved. The bottlenecksinclude:-

(a) the cost of Q' may be too high that the sum of the independent optimal statesis still the global optimal state;

(b) There may be no possibility at all to have sharable components and thereforea search for sharable components is wastage of resources.

(c) The new query plans may have a lower resource requirement than the previousone but when the resources taken to identify the plan (search cost) take onmore resources than the trade off hence no net saving on resources. 


\section{MODEL OF A COST-BASED QUERY OPTIMIZER}

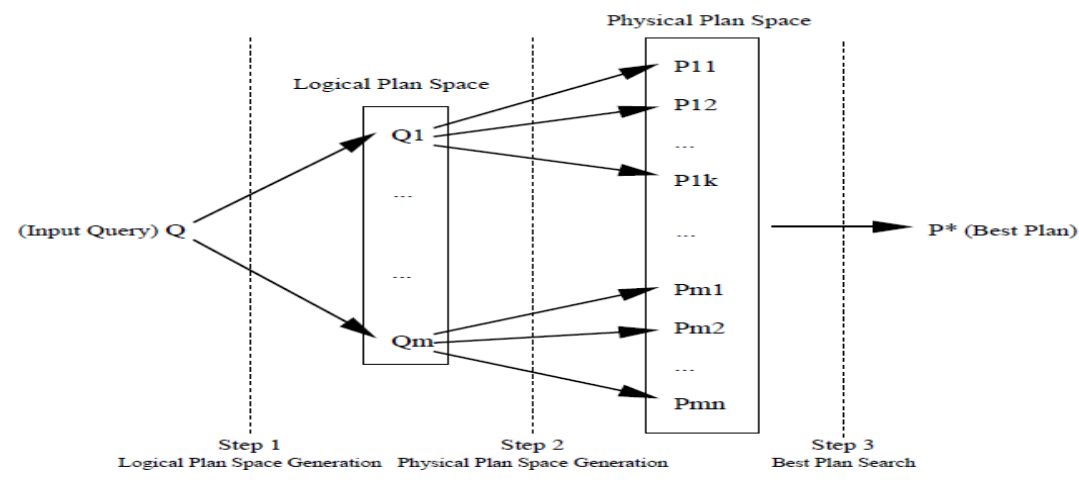

Figure 2: Overview of Cost-based Query Optimization

Figure gives an overview of the optimizer. Given the input query, the optimizer works in three distinct Steps:

3.1. Generate all the semantically equivalent rewritings of the input query.

In Figure Q1,......,Qm are the various rewritings of the input query Q. These rewritings recreated by applying "transformations" on different parts of the query; a transformation gives an alternativesemantically equivalent way to compute the given part. For example, consider the query $(A \bowtie(B \bowtie C))$. The join commutativetransformation says that $(B \bowtie C)$ is semantically equivalentto $(C \bowtie B)$, giving $(A \bowtie(C \bowtie B))$ as a rewriting. An issue here is how to manage the application of the transformation so as to guarantee that allrewritings of the query possible using the given set of transformations are generated, in as efficientway as possible. For even moderately complex queries, the number of possible rewritings can be very large. So,another issue is how to efficiently generate and compactly represent the set of rewritings.

\subsection{Generate the set of executable plans for each rewriting generated in the first step.}

Each rewriting generated in the first step serves as a template that defines the order in which thelogical operations (selects, joins, and aggregates) are to be performed - how these operations are to beexecuted is not fixed. This step generates the possible alternative execution plans for the rewriting.

For example, the rewriting $(A \bowtie(C \bowtie B))$ specifies that $A$ is to be joined with the result of joiningC with $B$. Now, suppose the join implementations supported are nested-loops-join, merge-join andhash-join. Then, each of the two joins can be performed using any of these three implementations, giving nine possible executions of the given rewriting.In Figure $\mathrm{P} 11, \ldots \ldots ., \mathrm{P} 1 \mathrm{k}$ are the $\mathrm{k}$ alternative execution plans for the rewritingQ1, and $\mathrm{Pm} 1, \ldots . .$, Pmnare the $\mathrm{n}$ alternative execution plans for Qm. The issue here, again, is how to efficiently generate the plans and also how to compactly store theenormous space of query plans.

3.3. Search the plan space generated in the second step for the "best plan".

Given the cost estimates for the different algorithms that implement the logical operations, the costof each execution plans is estimated. The goal of this step is to find the plan with the minimum cost.Since the size of the search space is enormous for most queries, the core issue here is how to performthe search efficiently. The Volcano search algorithm is based on top-down dynamic programming("memoization") coupled with branch-and-bound.

\subsection{Directed Acyclic Graph (Dag)}

We present an extensible approach for the generation of DAG-structured query plans.A Logical Query DAG (LQDAG) is a directed acyclic graph whose nodes can be divided into equivalence nodes and operation nodes; the equivalence nodes have only operation nodes as children and operationnodes have only equivalence nodes as children.

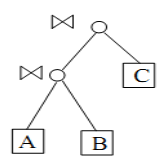

(a) Initial Query

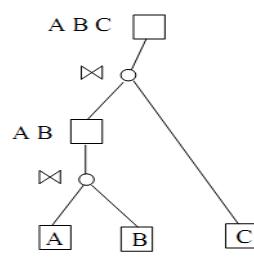

(b) DAG representation of query

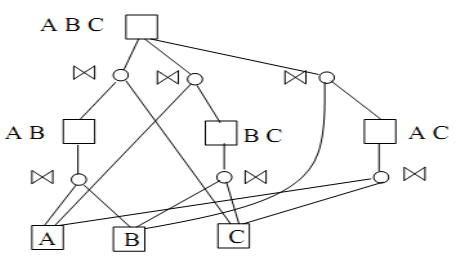

Commutativity not shown - every join node has another join node with inputs exchanged, below the same equivalence node) 


\section{HEURISTIC BASED ALGORITHMS}

4.1 The Basic Volcano Algorithm

This determines the cost of the nodes by using a depth first traversal of the DAG.The cost of operational and equivalence nodes are given

bycost $(\mathrm{o})=$ cost of executing (o) $+\sum$ ei $\in \operatorname{children}(\mathrm{o}) \operatorname{cost}(\mathrm{ei})$

and the cost of an equivalence node is given by

$\operatorname{cost}(\mathrm{e})=\operatorname{in}(\operatorname{cost}(\mathrm{oi}) \mid \mathrm{o} 2 \mathrm{children}(\mathrm{e})$

If the equivalence node has no children, then $\operatorname{cost}(\mathrm{e})=0$. In case a certain nodehas to be materialized, then the equation for $\operatorname{cost}(\mathrm{o})$ is adjusted to incorporatematerialization. For a materialized equivalence node, the minimum between thecost of reusing the node and the cost of recomputing the node is used. The equation therefore becomes

$\operatorname{cost}(\mathrm{o})=\operatorname{cost}$ of executing (o) $+\sum$ ei $\in \operatorname{children}(\mathrm{o}) \mathrm{C}(\mathrm{ei})$

whereC $(e i)=\operatorname{cost}(e i)$ if ei $! \in M$, and $=\min (\operatorname{cost}(e i)$, reusecost $(e i))$ ifei $\in M$.

\subsection{Volcano Algorithm}

PROCEDURE: Volcano(eq)

Input: Root node of Expanded DAG

Output: Optimized plan

Step 1: For every non-calculated op $\in$ child (eq)

Step 2: For every inpEqE child (op)

Step 3: Volcano(inpEq)

Step 4: If inpEq $\in$ leaf node

Step 5: $\operatorname{cost}(\mathrm{inpEq})=0$

Step 6: $\operatorname{cost}(\mathrm{op})=$ cost of executing $(\mathrm{op})+\sum \operatorname{Cost}(\mathrm{inpEq})$

Step 7: $\quad \operatorname{cost}(\mathrm{eq})=\min \{\operatorname{cost}(\mathrm{op}) \mid \mathrm{op} \in \quad$ children $(\mathrm{eq})\}$

Step 8: $\quad$ mark op as calculated

4.3 The Volcano SH Algorithm

In Volcano-SH the plan is first optimized using the Basic Volcano algorithm andthen creating a pseudo root merges the Basic Volcano best plans. The optimal queryplans may have common sub-expressions which need to be materialized and reused.

\subsection{The Volcano-RU Algorithm}

The Volcano-RU exploits sharing well beyond the optimal plans of the individualqueries. Though volcano SH algorithm considers sharing, it does it on only individuallyoptimal plans therefore some sharable components which are in sub-optimalplans are left out. Including sub-optimal states however implies that the samplespace of the nodes has to increase. The search algorithm must be able to put it intoconsideration so that the searching cost is still below the extra savings made.

\subsection{Calculation of Cost}

The nested loop algorithm works by reading one record from one relation, the outer relation, and passing over each record of the outer relation, the inner relation, joining the record of the outer relation with all appropriate records of the inner relation. The next record from the outer relation is then read and the whole of the inner relation is again scanned, and so on. The nested block algorithm works by reading a block of records from the outer relation and passing over each record of the inner relation(also read in blocks), joining the records of the outer relation with those of the inner relation. Historically, as much of the outer relation is read as possible on each occasion. If there are B pages in the memory, B-2 pages are usually allocated to the outer relation, one to the inner relation, and one to the result relation. For the mathematical model for cost estimation we tabulate the parameters as shown in the table.

Table No. 1 Variable Names with their Meaning

\begin{tabular}{|l|l|}
\hline Notation & Meaning \\
\hline V1 & Number of pages in relation R1 \\
\hline V2 & Number of pages in relation R2 \\
\hline $\mathbf{V}_{\mathbf{r}}$ & Number of pages in result of joining relation R1 and R2 \\
\hline $\mathbf{B}$ & Number of pages in memory for the use in buffers \\
\hline B1 & Number of pages in memory for relation R1 \\
\hline B2 & Number of pages in memory for relation R2 \\
\hline $\mathbf{B}_{\mathbf{R}}$ & Number of pages in memory for result \\
\hline
\end{tabular}


We denote the time taken to perform an operation $\mathrm{x}$ as $\mathrm{Tx}$. Each operation is a part of one of the join algorithms, such as transferring a page from disk to memory, or partitioning the content of a page. Table below shows the default values used to calculate the results below, were based on a disk drive with $8 \mathrm{~KB}$ pages, an average seek time of $16 \mathrm{~ms}$, and which rotates at 3600 RPM.

\section{Table no. 2 Constant Variables}

\begin{tabular}{|l|l|l|}
\hline Notation & Meaning & Values \\
\hline $\mathbf{T}_{\mathbf{C}}$ & Cost of constructing a hash table per page in memory & 0.015 \\
\hline $\mathbf{T}_{\mathbf{K}}$ & Cost of moving the disk head to the page on disk & 0.0243 \\
\hline $\mathbf{T}_{\mathbf{J}}$ & Cost of joining a page with a hash table in memory & 0.015 \\
\hline $\mathbf{T}_{\mathbf{T}}$ & Cost of transferring a page from disk to memory & 0.013 \\
\hline
\end{tabular}

We assume that the cost of a disk operation, transferring a set of Vx disk pages from disk to memory, or from memory to disk, can be given by

$$
\mathrm{Cx}=\mathrm{T}_{\mathrm{K}}+\mathrm{Vx} \mathrm{T}_{\mathrm{T}}
$$

Using this equation, we can derive the cost of transferring a set of $\mathrm{Vx}$ disk pages from disk to memory, or from memory to disk, through a buffer of size $\mathrm{Bx}$. It is given by

$$
\mathrm{C}_{\mathrm{I} / \mathrm{O}}(\mathrm{Vx}, \mathrm{Bx})=[\mathrm{Vx} / \mathrm{Bx}] \mathrm{Tk}+\mathrm{VxT}_{\mathrm{T}}
$$

We assume that the memory based part of the join is based on hashing. That is, a hash table is created from the pages of the outer relation, and the records of the inner relation are joined by hashing against this table to find records to join with.

As described above, the total available memory, B pages, is divided into a set of pages for each relation, B1, B2 and $B_{R}$. The general constraints that must be satisfied are:

- The sum of the three buffer areas must not be greater than the available memory: $\mathrm{B} 1+\mathrm{B} 2+\mathrm{B}_{\mathrm{R}}<=\mathrm{B}$.

- The amount of memory allocated to relation R1 should not exceed the size of relation $\mathrm{R} 1$ : $1<=\mathrm{B} 1<=\mathrm{V} 1$.

- The amount of memory allocated to relation R2 should not exceed the size of relation $\mathrm{R} 2: 1<=\mathrm{B} 2<=\mathrm{V} 2$.

- Some memory must be allocated to the result: $B_{R}>=1$ if $V_{R}>=1$.

As described above, $\mathrm{V} 1<=\mathrm{V} 2$, therefore relation $\mathrm{R} 1$ is the outer relation. It is read previously once, B1 pages at a time, in [V1/B1] I/O operations. Thus, relation R2 will be read [V1/B1] times, B2 pages at a time. Each pass over relation $\mathrm{R} 2$, except the first, reads V2-B2 pages due to the rocking over the relation. The total cost of nest block join is given by-

Cost of transferring a set V1 pages through buffer of size B1:

$$
\mathrm{C}_{\text {Read R } 1}=\mathrm{C}_{\mathrm{I} / \mathrm{O}}(\mathrm{V} 1, \mathrm{~B} 1)
$$

Cost of creating Hashed Pages from V1 pages:

$$
\mathrm{C}_{\text {Create }}=\mathrm{V} 1 \mathrm{~T}_{\mathrm{C}}
$$

Cost of transferring V2 pages through buffer of size B2:

$\mathrm{C}_{\text {Read R2 }}=\mathrm{C}_{\mathrm{I} / \mathrm{O}}(\mathrm{V} 2, \mathrm{~B} 2)$

Cost of joining each hashed page with $\mathrm{V} 2$ pages

$$
\mathrm{C}_{\text {Join }}=\mathrm{V}_{2} \mathrm{~T}_{\mathrm{J}}
$$

Cost of Writing back the result into the disk drive:

$$
\mathrm{C}_{\text {Write RR }}=\mathrm{C}_{\mathrm{I} / \mathrm{O}}\left(\mathrm{V}_{\mathrm{R}}, \mathrm{B}_{\mathrm{R}}\right)
$$

Total Cost of Operation:

$$
\begin{aligned}
\mathbf{C}_{\mathrm{NB}}= & \mathbf{C}_{\text {Read R1 }}+\mathbf{C}_{\text {Create }}+\mathbf{C}_{\text {Read R2 }}+\mathbf{C}_{\mathrm{Join}} \\
& +\mathbf{C}_{\text {Write RR }}
\end{aligned}
$$

\section{RESULTS}

The goal of the basic experiments was to quantify the benefits and cost of the three heuristics for multi-query optimization, Volcano-SH, Volcano-RU and Greedy, with plain Volcano-style optimization as the base case. We used the version of Volcano-RU which considers the forward and reverse orderings of queries to find sharing possibilities, and chooses the minimum cost plan amongst the two. Figure 3 shows the comparisons of different algorithms. 


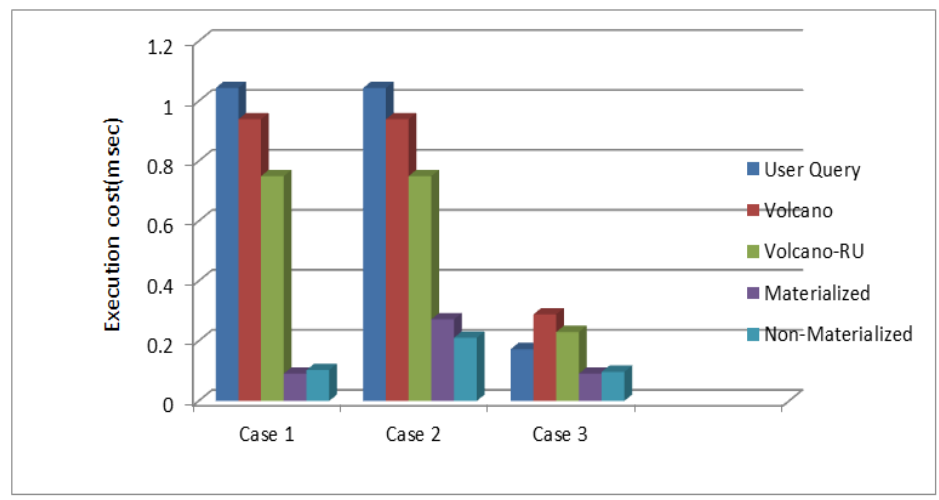

Figure 3: Comparison of different algorithms.

\section{FUTURE WORK}

Execution cost is minimum in greedy algorithm and is maximum in Volcano is maximum whereas the optimization cost is maximum in greedy algorithm and minimum in volcano. Thus from this study there is scope in future to combine these two algorithm so that the execution cost of greedy and optimization cost from volcano can be used to evaluate best optimization results for Multi-query optimization.

Since we worked on heuristic based algorithm we just consider only transfer time. for future to work on real time database the seek time and latency time should be considered.

\section{CONCLUSION}

The benefits of multi-query optimization were also demonstrated on a real database system. Our implementation demonstrated that the algorithms can be added to an existing optimizer with a reasonably small amount of effort. Our performance study, using queries based on the TPC-D benchmark, demonstrates that multi-query optimization is practical and gives significant benefits at a reasonable cost. The greedy strategy uniformly gave the best plans, across all our benchmarks, and is best for most queries; Volcano-RU, which is cheaper, may be appropriate for inexpensive queries.

Thus we can conclude that the techniques of using Volcano heuristic algorithms are the best approach for Multi-QueryOptimization as compared to other optimization techniques and are practically well implemented. And the comparative study shows that the Volcano, Volcano-SH andVolcano-RU give different optimized results at different cases depending on the logically equivalent queries.

\section{REFERENCES}

[1] [RSR+99] Prasan Roy, PradeepShenoy, KrithiRamamritham, S. Seshadri, and S. Sudarshan. Don't trash your intermediate results, cache 'em. Submitted for publication, October 1999.

[2] [RSS96] Kenneth Ross, DiveshSrivastava, and S. Sudarshan. Materialized view maintenance and integrity constraint checking: Trading space for time. In SIGMOD Intl. Conf. on Management of Data, May 1996.

[3] Thomas Neumann and Guido Moerkotte. An e_cient framework for order optimization. In Proceedings of the 20th International Conference on Data Engineering, 30 March - 2 April 2004, Boston, MA, pages 461-472. IEEE Computer Society, 2004.

[4] [CCH+98] Latha Colby, Richard L. Cole, Edward Haslam, NasiJazayeri, Galt Johnson, William J. McKenna, Lee Schumacher, and Da vid Wilhite. Redbrick Vista: Aggregate computation and management. In Intl. Conf.on Data Engineering, 1998.

[5] SurajitChaudhuri, Ravi Krishnamurthy, Spyros Potamianos, AndKyuseok Shim. Optimizing queries with materialized views. In Intl. Conf. on Data Engineering, Taipei, Taiwan, 1995.

[6] SurajitChaudhuri and VivekNarasayya. An efficient cost-driven index selection tool for microsoft SQL Server. In Intl. Conf. Very Large Databases, 1997.

[7] [GHRU97] H. Gupta, V. Harinarayan, A. Rajaraman, and J. Ullman. Index selection for olap. In Intl. Conf. on Data Engineering, Binghampton, UK, April 1997.

[8] Arjan Pellenkoft, Cesar A. Galindo-Legaria, and Martin Kersten. The Complexity of Transformation-Based Join Enumeration. In Intl. Conf. Very Large Databases, pages 306-315, Athens, Greece, 1997. 